\title{
Curiosity: A Behavioral Biology Perspective
}

\author{
Coltan Scrivner ${ }^{1,2}$
}

Email: cscrivner@uchicago.edu

${ }^{1}$ Department of Comparative Human Development, The University of Chicago

${ }^{2}$ Institute for Mind and Biology, The University of Chicago

\begin{abstract}
Since Berlyne's groundbreaking work in the 1960's, curiosity has been a popular topic for psychological research. Despite a rich history of research, scientists have not been able to agree upon a single definition or taxonomy of curiosity. These diverging perspectives have led to a breadth of research that has yet to be integrated under one framework. Moreover, most research on curiosity has focused on neural mechanisms and ontogenetic characteristics, while the evolutionary aspects of curiosity have received little attention. I propose that research on curiosity can benefit from an evolutionary perspective, and more broadly from a biological perspective on information-gathering behavior. In this chapter, I synthesize the literature on curiosity from the perspective of behavioral biology - i.e., Tinbergen's four questions. The behavioral biology framework provides a powerful lens through which questions about behavior can be asked and iterative empirical work and theoretical construction can take place. In particular, I argue that evolutionary perspectives on curiosity can help identify the "joints" of nature at which curiosity may be carved. By identifying the function of different types of curiosity, a more robust and universal taxonomy of curiosity can be created.
\end{abstract}

Keywords: Curiosity; Ethology; Evolution 


\section{Overview of Perspectives on Curiosity}

Like many aspects of human psychology that seem too obvious to require a definition and rigorous scientific explanation, curiosity has been difficult to define and explain. William James (1890) proposed that there are two types of curiosity. The first involves seeking information about novel objects. This type of curiosity, James contends, is present in most animals. The second type, which James suggested has almost nothing to do with the first and is specific to humans, is scientific curiosity or metaphysical wonder. James argued that this type of curiosity is more concerned with ways of conceptualizing objects rather than the objects themselves. Scientific curiosity responds not to novel stimuli per se, but rather to gaps in knowledge. Still, James only briefly mused about curiosity, and did not offer empirical evidence for his conceptual distinction. Daniel Berlyne (1954) was the first experimental psychologist to investigate curiosity in detail. He also broke down curiosity into two major types that results in a distinction that resembled James's. The first type of curiosity he called perceptual curiosity, referring to the drive to gather information about novel stimuli until they are no longer so novel. Berlyne gave the example of a rat that exhibits increased exploratory activity around a new stimulus. $\mathrm{He}$ distinguished this novelty-seeking form of curiosity from epistemic curiosity, which he suggests is more about acquiring knowledge and is largely unique to humans.

Berlyne (1966) later divided curiosity along a second axis: specific vs diversive. Specific curiosity occurs when there is a gap in information about some phenomenon. This gap produces discomfort, which motivates one to seek out additional information about that specific phenomenon. An example of this can be seen if you briefly show a participant a complex image. Given the chance, the participant will most likely want to re-investigate the image, reducing the gap in knowledge that was produced from the momentary look. On the other hand, diversive curiosity arises when an organism is under-stimulated and seeks novel stimulation to satisfy the feeling of deprivation. An example of this can be seen if a participant is placed in a dark room with the option to press buttons to make lights appear. The human participant will press buttons in a sequence that produces variety in the light patterns (Berlyne, 1966).

Decades later, Loewenstein (1994) offered a reinterpretation of the psychology of curiosity. He criticized the concept of diversive curiosity, arguing that it was more akin to sensation seeking 
and related to boredom than it was to scientific curiosity. He noted that previous theories of curiosity fail to answer why people like to feel curious and, if they do indeed like it, why they try to end it through information-seeking. Loewenstein offers a new perspective on curiosity in an attempt to reconcile these issues with previous theories. His information-gap theory of curiosity, which deals only with internally motivated, specific state curiosity (as opposed to externally motivated, diversive, or trait curiosity), predicts that curiosity arises to resolve uncertainty about a specific topic. The logical conclusion of this prediction is that curiosity will have an inverted-U shape relationship with knowledge such that curiosity rises as an individual learns about a topic, peaks when an individual possesses a moderate degree of knowledge or confidence in a topic, and declines thereafter.

Several other theoretical frameworks have been offered, such as curiosity as a "knowledge emotion" (Silvia, 2010) or curiosity functioning to maximize the ability to make good decisions in the future (Dubey \& Griffiths, 2020a; Silvia, 2010). Each of these frameworks has its own set of empirical studies that support the main theory, making it difficult to ascertain which framework is most useful. One aspect that is missing from most previous theoretical frameworks of curiosity is an evolutionary perspective. While some do offer "functional" explanations for curiosity, these functional explanations still raise the question: why does this state or trait exist? A proposed answer to this "why" question is important for guiding research on the psychology of curiosity.

\section{The Behavioral Biology Framework}

I propose in this chapter that research on curiosity can benefit from an evolutionary perspective, and more broadly from a biological perspective on information-gathering behavior. One framework that is likely to be illuminating for research on curiosity is the framework proposed by ethologist Nikolaas Tinbergen for investigating behavior from a biological perspective. Tinbergen (1963) proposed that to fully understand a behavior, four types of questions must be asked. The first question is about the proximate causation or mechanism underlying the behavior. In other words, what is the most immediate cause of the behavior? This can be in terms of either the stimulus that evokes the behavior or in the information processing that occurs in the brain (though a comprehensive mechanistic explanation will address both). The 
second question asks how the behavior changes over time during the lifespan of the organism i.e., the developmental or ontogenetic question. The third question relates to the adaptive value of the behavior. What is the function of the behavior and how does this relate to survival and/or reproduction? The fourth question is the phylogenetic question. How did this behavior evolve over time? Is it present in closely related species in one form or another?

These four questions split behavioral explanations into two kinds: proximate and ultimate. Mechanistic explanations and developmental explanations are proximate explanations. Proximate explanations focus on 'how' - how a behavior works and how it changes throughout the life-course. Adaptive (or functional) and phylogenetic explanations are ultimate explanations. These explanations focus on 'why' - why does a behavior manifest the way that it does and why does it exist in the species? These four questions are meant to offer a guide for a robust biological investigation of a particular behavior. While others have suggested that curiosity be investigated from an ethological perspective (Kidd \& Hayden, 2015), this perspective has not been widely adopted in research on the psychology of curiosity. Moreover, research and theoretical accounts on the adaptive significance of curiosity are severely lacking from the psychological literature.

There appears to be no agreed-upon definition for curiosity, and this chapter will not argue for one definition over another. Some definitions include all exploratory behavior (e.g., specific and diversive curiosity) and some exclude extrinsically motivated behavior. Using a more specific definition of curiosity that only includes internally motivated behavior can provide direction and a necessary narrowing of research, but it comes with its own problems. In particular, it is difficult to identify the motives behind particular curiosity-like behaviors in non-human animals (Kidd \& Hayden, 2015). Rather than circumscribe curiosity more stringently, this chapter will take a broad look at information-seeking behavior as at least analogous to curiosity. This is a useful approach under the evolutionary paradigm because it allows connections to be drawn between human curiosity and its analogs in other animals. This perspective is especially important for the phylogenetic and functional explanations of curiosity. Thus, the general ethological definition of curiosity as a drive state for information will be used here, as it was in Kidd \& Hayden's (2015) analysis of curiosity from the ethological paradigm. A robust analysis 
of information-seeking utilizing Tinbergen's four questions may allow for an evolutionarilyinformed taxonomy of curiosity to be created and tested in future research.

\section{Phylogenetic Level}

Anyone with a pet at home is likely to agree that animals can exhibit curiosity. Dogs are insatiably curious about new toys that are brought home, and cats will thoroughly inspect all grocery bags if given the opportunity. Glickman and Sroges (1966) conducted the seminal experimental investigation of animal curiosity in over 200 animals at two zoos in Chicago and New York City. In particular, the study assessed the degree to which different animals would investigate novel objects. Glickman and Sroges introduced to the cages where the animals were housed a pair of wooden blocks, two lengths of steel chain, two pieces of wooden dowel, two pieces of rubber tubing, and a paper ball. Where possible, males and females of the same species were tested and items were either not reused or were thoroughly washed before reuse. Each item was placed in the cage for six minutes, with the session divided into five-second intervals. Within each interval, orienting behavior (looking) and contact was assessed. Glickman and Sroges found that primates and carnivores were the most curious animals in the sample, while rodents, "primitive mammals," and reptiles appeared significantly less curious about the novel objects. Juveniles across species were also more curious than adults.

It is clear from the zoo study that most mammals exhibited something like curiosity. Glickman and Sroges interpreted their findings in light of the feeding patterns and predators in the animals' natural habitats. In other words, animals whose natural habitats offer more refuge from danger and require more complex behaviors for food acquisition might display greater specific curiosity in response to novel objects.

The increased neophilic behavior and genetic similarity of non-human primates to humans make them a prime point of comparison for questions about the phylogenetic roots of human-like curiosity. Like humans, monkeys demonstrate specific curiosity about information when solving puzzles, even in the absence of clear extrinsic motivation (Harlow, 1950; Harlow et al., 1950). Even in accordance with a stricter definition of curiosity, monkeys demonstrate curiosity about counterfactuals by sacrificing reward to learn information with no strategic benefit (Wang \& 
Hayden, 2019). Even though species-typical tendencies toward curiosity-like traits exist, individuals within a species also vary with respect to curiosity due to a combination of genetic predispositions and environmental cues (Sih et al., 2004). For example, squirrel monkeys who experience mild stress early in their lives are more likely to exhibit increased novelty-seeking behaviors (Parker et al., 2007). Differences between conspecifics in exploratory behavior can be large, and have been linked to reproductive fitness (Reader, 2015).

It is currently unclear how similar curiosity in humans is to curiosity in non-human animals. Certainly novelty-seeking and exploratory behavior can be closely compared both theoretically and empirically. However, curiosity about things like causation may be more difficult to compare for a phylogenetic analysis. Still, various kinds of curiosity-like behavior, broadly speaking, have been documented in non-human animals and may be mappable onto human traits (Gosling \& John, 1999). Likewise, these various aspects of curiosity may be connected through an evolutionarily ancient neurocircuitry system that is largely conserved in mammals (Montag \& Panksepp, 2017; Wright \& Panksepp, 2012). A more complete cataloguing of curiosity-like behaviors in non-human animals may help shine a light on what is unique about human curiosity (if anything). A functional evolutionary analysis of curiosity may help guide phylogenetic analyses in an iterative manner.

\section{Functional (Adaptive) Level}

Berlyne offered one of the first functional accounts of curiosity in the psychological literature when he argued that part of the function of the curiosity drive was to resolve uncertainty (Berlyne, 1960). Curiosity as uncertainty reduction has remained a popular idea and has been an important part of many curiosity research programs (Kashdan et al., 2018; Litman \& Jimerson, 2004; Loewenstein, 1994). While it seems reasonable that reducing uncertainty about the world is a key feature of curiosity, surely this is not the only feature of curiosity. If this were the case, it would be difficult for organisms to focus their curiosity in a useful fashion. After all, uncertainty exists about nearly every phenomenon. In some cases, curiosity may be driven by extreme uncertainty, while in others it is driven by moderate uncertainty (Dubey \& Griffiths, 2020a). At best, the argument that the function of curiosity is to reduce uncertainty is incomplete and leaves much to be desired. 
Dubey and Griffiths (2020b) have posited that the function of curiosity is to increase the usefulness of one's own knowledge. Under this hypothesis, the value of knowledge is a function of the organism's current knowledge about the environment and the probability of encountering some stimulus in the future. In positing this function, Dubey and Griffiths have attempted to reconcile the uncertainty-reduction perspective and the novelty-seeking perspective on curiosity. When an organism is faced with a novel stimulus, it should investigate it only if that stimulus is likely to occur again. Still, this raises the question of how some stimulus in the environment might be perceived and integrated in the mind. For example, the current value of some piece of information may be at odds with the value of that information over the course of human evolution. Most people in the US do not encounter wild snakes in their lifetime. However most people in the US still display an attentive bias toward snakes (LoBue \& DeLoache, 2008; Öhman $\&$ Mineka, 2003). If this attentive bias falls under the purview of curiosity, then the "value" the knowledge appears to be influenced by a deep evolutionary history with the stimulus. To account for this type of curiosity, "current knowledge" in the Dubey \& Griffiths hypothesis must include some calculation of the attentive predispositions that humans have inherited from their ancestors. Likewise, most people do not encounter serial killers in their lifetime, yet true crime is a wildly popular form of entertainment. Many people are genuinely curious about serial killers, despite the fact that the probability of encountering a serial killer is exceedingly low. Thus, something other than just current knowledge about the environment and the probability of encountering some stimulus in the future is influencing curiosity.

Part of the reason why a taxonomy of curiosity has not been agreed upon is that the function of curiosity is not agreed upon. Of course, it can be difficult to give a function to a concept that is ill-defined. One way to approach this is to try to identify the functions of the various behaviors that constitute the umbrella construct of curiosity. Many of these behaviors may well be related to individual success and reproductive fitness. Because of this, the psychological mechanisms that mediate curiosity have probably been shaped by natural selection. Consequently, these psychological mechanisms will be well-suited for dealing with particular socio-ecological problems. Identifying these socio-ecological problems in conjunction with a comparative 
psychological analysis (i.e., the phylogenetic analysis), may shed light on an appropriate taxonomy for curiosity.

Curiosity has been classified in many different ways. For example, James classified curiosity as either novelty-seeking or scientific curiosity, while Berlyne split curiosity along the specific/diversive axis and the perceptual/epistemic axis. Litman (2008) split epistemic curiosity into interest and deprivation based on whether it stimulated positive affect and diversive exploration (interest) or reduced uncertainty and stimulated specific exploration (deprivation). More recently, Kashdan et al. (2018) constructed the five-dimensional curiosity scale to try to capture the bandwidth of curiosity. Through a series of studies that began with over 100 curiosity-related items, Kashdan et al. identified five major dimensions of curiosity: joyous exploration, deprivation sensitivity, stress tolerance, social curiosity, and thrill seeking. A more recent iteration of the scale splits social curiosity into overt and covert social curiosity (Kashdan et al., 2020). While the new scale from Kashdan et al. provides a data-driven approach to identifying dimensions of curiosity, it leaves some important theoretical questions about curiosity unanswered. For example, are these different dimensions caused by the same underlying suite of psychological mechanisms? What is the function of curiosity in each of these dimensions and why might they be divided this way?

Evolutionary psychologists have argued that emotions can be understood as coordinating mechanisms that process particular kinds of information and regulate other aspects of physiology, behavior, and cognition (Al-Shawaf et al., 2015; Cosmides \& Tooby, 2000). Under this view, "emotions" include emotions as they have been traditionally understood (e.g., fear, disgust, anger) as well as motivational states and drives such as lassitude (Schrock et al., 2020) and hunger (Al-Shawaf, 2016). Viewing emotions, motivational states, and drives as coordinating mechanisms has several advantages. It offers a form of non-arbitrary emotion classification, increased heuristic value for identifying emotions that should be studied, and a systematic method for a priori hypothesis generation and testing through evolutionary task analysis (Al-Shawaf et al., 2015; Marr, 1982). Evolutionary task analysis involves asking four key questions: (1) what adaptive problem, if any, did this mechanism evolve to solve? (2) which subtasks must be solved in the solution of this adaptive problem? (3) which information- 
processing programs are capable of solving these subtasks? and (4) how should these programs be coordinated to deliver a well-designed solution to this adaptive problem? (Al-Shawaf et al., 2015). Studying curiosity through this functional lens may offer several avenues for future research and help create a more effective and functionally accurate taxonomy of curiosity.

One type of curiosity that does not seem to be captured in previous work is morbid curiosity. Morbid curiosity has been defined as a motivation to seek out information about threatening or dangerous phenomena (Scrivner, 2020). People high in morbid curiosity may be more likely to expose themselves to simulations of danger through fiction and may learn particular emotional or behavioral strategies from exposure to real or fictionalized dangerous situations (Scrivner, 2021; Scrivner et al., 2021). Moreover, morbid curiosity seems to be an individual difference that is distinct from the five dimensions of curiosity, disgust sensitivity, and sensation-seeking (Oosterwijk, 2017; Scrivner, 2020).

A clear function of morbid curiosity also appears to be emerging. Dangerous situations have been and continue to be present in everyday life for humans. In general, an organism should try to avoid dangerous situations. However, there is some value in knowing about danger, as this could lead to a better ability to avoid or deal with these situations in the future. Due to the unique ability to simulate experiences through fiction, humans can create low-cost learning "experiences" with danger. These situations can be quite immersive, activating physiological, behavioral, and emotional responses that correspond to what would occur if the situation were real (Andersen et al., 2020). This allows those engaging with recreational fear to learn more about the situation, how they would respond, and how they can improve that response for a better outcome. Some preliminary evidence suggests that engagement with frightening fiction could correspond to psychological resilience in the face of situations that induce anxiety or feelings of uncertainty (Scrivner et al., 2020b). However, because these situations can be scary, disgusting, or otherwise off-putting, they require some motivation to simulate. Morbid curiosity provides the motivational boost that shifts the perceived cost/benefit ratio to encourage approach behavior that leads to engagement with morbid fictional material. 
My own research on the psychology of morbid curiosity was inspired by the fact that dangerous situations are common, that these situations can have fitness consequences, that humans can simulate these dangerous situations, and that learning about these situations can lead to more efficient responses. In other words, a functional approach was taken in the development of this research program. Similar arguments were made for the inclusion of a social curiosity dimension in the five-dimensional curiosity scale. Kashdan et al. (2018) argued that being curious about people warranted further investigation as a separate dimension due to the primacy of interpersonal relationships in human life. These kinds of functional approaches to curiosity can offer specific areas in which to investigate behavior in humans and other animals and may help produce a reliable taxonomy of curiosity.

\section{Developmental Level}

As is the case with pet owners noticing the curiosity of their animals, parents would most likely agree that children display a great deal of curiosity. Human infants in particular have a profound proclivity for exploring and learning about their environment. Infants possess particular attention biases that facilitate learning. Some of these include biases that promote motion detection (Agyei et al., 2016), shape or object learning (Yee et al., 2012), and attention to faces (Farroni et al., 2005). Similar to predispositions in language acquisition, these simple biases guide infant perception so that they can more efficiently learn about the important aspects of a world with a nearly infinite number of stimuli.

Some research on infant learning supports both complexity-based preferences proposed by Berlyne (1960) and information-gap motives proposed by Loewenstein (1994). According to these models, learners should prefer intermediate levels of complexity or moderate information gaps. Material in this range would fall into an optimal level of understanding for productive and efficient learning. Indeed, infants do seem to prefer both visual and auditory stimuli that present a moderate level of complexity (Kidd et al., 2012, 2014). This finding is in line with Piaget's (1945) argument that play serves to construct knowledge through interactions with the environment. In this model, curiosity is the motivation that drives the behavior (play). This also corresponds with Csikszenthmihalyi's (1990) notion of flow, wherein an inverted U-shaped relationship exists between perceived challenge of some task and intrinsic motivation. As with 
play and curiosity, moderately challenging tasks offer an opportunity for optimal performance.

In addition to children being voracious learners, the juvenile period in humans is exceptionally long and costly. Children require extensive care and may not be self-sufficient until well into their teenage years. Humans in forager societies consume nearly $25 \%$ of their lifetime energy consumption by age 15 , yet only produce about $5 \%$ of their anticipated energy acquisition by the same age (Kaplan et al., 2000). This incredible imbalance in energy consumption and acquisition suggests that the extended juvenile period in humans is probably adaptive in some ways. Given the complex nature of human societies and human psychology, human childhood likely needs to be extended in order to allow humans time to develop and prepare for the uniquely complex adult human life.

Thus, extended childhood appears to be a human life-history adaptation. Alison Gopnik (2020) has argued that extended childhood in humans is a solution to the explore-exploit problem. The explore-exploit problem refers to the decisions that organisms have to make when they are faced with the opportunity to either exploit known aspects of their environment for resources or rewards, or to explore unknown aspects of their environment for a payoff that could be large, small, or non-existent. Moreover, the explore decision may not provide current payoff, but may instead provide future payoff. Because natural environments are so complex and time and energy are limited, organisms cannot possibly try all solutions for the best one. Most solutions to the explore-exploit dilemma begin with exploration and gradually move toward exploitation. Once enough is known about the environment, it becomes more efficient and productive to exploit for current benefits rather than to explore for unknown benefits or future benefits.

Gopnik and her colleagues have argued that this solution is also represented in the developmental trajectory of humans (Gopnik et al., 2015, 2017; Lucas et al., 2014). Humans have an unusually long juvenile period in which exploration and learning is prioritized over exploitation. Although the human juvenile period is particularly well-adapted for exploration, the juvenile period of other animals, and certainly other primates, may also suit this purpose. Comparative evidence suggests that juveniles of many species learn better, explore more options, and are often more innovative than adults, a finding that holds in mice (Johnson \& Wilbrecht, 2011), birds (O'Hara 
\& Auersperg, 2017), hyenas (Benson-Amram \& Holekamp, 2012), and monkeys (Bergman \& Kitchen, 2009; Perry et al., 2017). Even among adults, increased innovation and diverse exploration of hypotheses is associated with younger age. For example, theoretical scientists, whose work focuses on re-envisioning how something works, tend to produce their best and most innovative work earlier in their career (Scrivner \& Maestripieri, 2018). Indeed, similarities between the cognition of children and theoretical scientists have been noted elsewhere (Gopnik, 1996). Thus, the juvenile period as a period of exploration is a general principle that applies especially strongly to humans. Given the complexity of human social life and the ability to forecast and imagine hypothetical and counterfactual scenarios, it is no surprise that human children are exceptionally curious and motivated learners.

\section{Mechanistic Level}

The most proximate cause, or the mechanism, of all human behavior is rooted in the brain. Dopamine plays a key role in the mechanistic basis of curiosity. Dopamine is a neurotransmitter that modulates motivational behavior, in particular learning and reward-related motivation. Dopaminergic activity, particularly in the midbrain dopaminergic neurons, appears to be relevant for curiosity. For example, participants in one study answered trivia questions and rated their curiosity for each answer while their brain activity was assessed via fMRI. When participants reported higher curiosity about answers to trivia questions, fMRI analyses revealed greater activity in the midbrain dopaminergic neurons (Gruber et al., 2014).

Though the exact relationship between dopamine and reward-related motivation is not entirely clear, one popular hypothesis is that dopaminergic neurons encode predictions errors in expected reward (Arias-Carrión et al., 2010). This neuroscientific hypothesis lines up well with the psychological hypothesis that curiosity functions to minimize uncertainty. By encoding prediction errors through dopaminergic activity, animals will be able to improve future predictions relating to the outcome of a particular reward-relevant stimulus. In turn, this leads to better decisions about how to act toward this reward-stimulus in the future. Thus, dopamine's 
role in moderating behavior by encoding prediction error may be one neurological mechanism underlying curiosity.

Some researchers have hypothesized that the brain normalizes and encodes information from a variety of sources into a common currency by which action decisions can be made (Levy \& Glimcher, 2012). By normalizing the value of information into a common currency, increasingly complex decisions can be made that include information from a variety of qualitatively different sources. If, per Dubey and Griffiths, the function of curiosity is to increase the value of knowledge, and value is neurologically normalized into a common currency, then information that is perceived as more important may be more likely to provoke curiosity. Dubey and colleagues (2019) tested this hypothesis in a study where they presented participants with scientific facts and manipulated how valuable information about those facts would be. As they predicted, participants rated their curiosity about a fact as higher when that fact was perceived to be more valuable. These findings correspond with the hypothesis that the function of curiosity is to learn useful information about the world and improve the value of knowledge that one holds.

However, humans are often curious about information that has no instrumental value (as are monkeys, e.g., Wang \& Hayden, 2019). Interestingly, the common neural code for informationrepresentation appears to encode both instrumental and non-instrumental information, casting doubt on the distinction that is sometimes made in curiosity between intrinsic and extrinsic motivation for information-seeking (Kobayashi \& Hsu, 2019). The common neurological activation for information-seeking also corroborates Panksepp's SEEKING system among mammals (Panksepp \& Moskal, n.d.). Moreover, these studies suggest that information may be neurologically valued in the same manner as other goods, a finding that has been previously demonstrated in monkeys (Bromberg-Martin \& Hikosaka, 2011).

While much research has been conducted on midbrain dopaminergic neurons, less work has looked at where curiosity "begins" in the brain. Curiosity-related input from the sensory organs may first be processed in the orbitofrontal cortex (OFC). Blanchard et al. (2015) conducted a study where monkeys could sacrifice a primary reward such as water for information about a gamble. Blanchard and colleagues found that while OFC neurons appeared to encode variables 
like water amount and value of the information about the gamble, integration of these variables into a common currency did not occur in the OFC. Instead, the researchers concluded that the OFC encoded the relevant variables before sending the signal downstream to the midbrain, where signals are integrated into the common currency for rewards.

\section{Conclusions}

Curiosity continues to be one of the most important and widely valued psychological traits. However, research on curiosity has been focused on narrowly defined definitions or taxonomies. In this chapter, I argue that one productive way for researchers to approach the study of curiosity is to utilize the behavioral biology framework. Tinbergen's four questions mechanism, development, evolutionary function, and phylogeny - allow for a broad, comprehensive analysis of animal behavior. Notably, Kidd \& Hayden (2015) have argued for the same approach to curiosity. By stepping back and incorporating a larger perspective on curiosity, psychologists can avoid the trap noted by Tinbergen in his 1963 paper, where he stated,

"It has been said that, in its haste to step into the twentieth century and to become a respectable science, Psychology skipped the preliminary descriptive stage that other natural sciences had gone through, and so was soon losing touch with the natural phenomena." (p. 411)

Science has come a long way since Tinbergen first made this claim, and psychologists, neuroscientists, biologists, and other researchers interested in studying curiosity have a wealth of new knowledge, tasks, instruments, and technology at their fingertips. Researchers studying curiosity should avoid being overly narrow in their description of curiosity and aim instead for an integration of perspectives and empirical data. To do this will require a broader perspective on curiosity, like the one offered by Tinbergen's four questions.

Analyzing curiosity through this perspective may also help produce a taxonomy for curiosity that carves nature at its joints. Curiosity has been split in several different ways, including noveltyseeking and scientific (James, 1890), perceptual and epistemic (Berlyne, 1954) specific and diversive (Berlyne 1966), interest and deprivation (Litman, 2008), forward and backward (Shin \& Kim, 2019), bottom-up and top-down (Kashdan, 2012), and along several other dimensions 
(e.g., Kashdan et al., 2020). One reason why so much diversity exists in classifying and defining curiosity is because functional accounts of curiosity have yet to be rigorously investigated. While some work has been done in robotics and artificial intelligence on the function of curiosity, these studies would benefit from a functional evolutionary analysis of human and animal curiosity.

Evolutionary perspectives may help identify the "joints" of nature at which curiosity may be carved (Sznycer et al., 2017). This can be assisted by comparative evidence from a variety of organisms, from simple creatures like C. elegans to phylogenetically similar primates like chimpanzees and bonobos. Identifying curiosity-like behaviors in other animals and understanding their development across the animal's lifespan can also shed light on the evolutionary function of these behaviors. The identification of curiosity-like behaviors and their development can lead to more informed mechanistic analyses. These mechanistic analyses can, in turn be carefully manipulated in the laboratory to test various hypotheses about curiosity and its function.

While these four questions can in principle be considered in isolation, it is often productive to consider them in groups. For example, one could look at how the neurobiological mechanisms that underlie some curiosity-like behavior change throughout the life-course. Change in curiosity-like behavior throughout the life-course could be compared in phylogenetic analysis, which could in turn provide information about the function of that particular behavior. Functional accounts of curiosity can be pitted against each other through mechanistic manipulations in the laboratory. Thus, the behavioral biology framework provides a powerful lens through which questions about behavior can be asked and iterative empirical work and theoretical construction can take place. 


\section{References}

Agyei, S. B., van der Weel, F. R. R., \& van der Meer, A. L. H. (2016). Development of Visual Motion Perception for Prospective Control: Brain and Behavioral Studies in Infants. Frontiers in Psychology, 7, 100.

Al-Shawaf, L. (2016). The evolutionary psychology of hunger. Appetite, 105, 591-595. https://doi.org/10.1016/j.appet.2016.06.021

Al-Shawaf, L., Conroy-Beam, D., Asao, K., \& Buss, D. M. (2016). Human emotions: An evolutionary psychological perspective. Emotion Review, 8(2), 173-186. https://doi.org/10.1177/1754073914565518

Al-Shawaf, L., \& Lewis, D. M. (2017). Evolutionary psychology and the emotions. In V. Zeigler-Hill and T. K. Shackelford (Eds.). Encyclopedia of personality and individual differences, 1-10.

Andersen, M. M., Schjoedt, U., Price, H., Rosas, F. E., Scrivner, C., \& Clasen, M. (2020). Playing With Fear: A Field Study in Recreational Horror. Psychological Science, 956797620972116.

Arias-Carrión, O., Stamelou, M., Murillo-Rodríguez, E., Menéndez-González, M., \& Pöppel, E. (2010). Dopaminergic reward system: a short integrative review. International archives of medicine, 3(1), 1-6. https://doi.org/10.1186/1755-7682-3-24

Benson-Amram, S., \& Holekamp, K. E. (2012). Innovative problem solving by wild spotted hyenas. Proceedings of the Royal Society B: Biological Sciences, 279(1744), 4087-4095.

Bergman, T. J., \& Kitchen, D. M. (2009). Comparing responses to novel objects in wild baboons (Papio ursinus) and geladas (Theropithecus gelada). Animal Cognition, 12(1), $63-73$.

Berlyne, D. E. (1954). A theory of human curiosity. British Journal of Psychology, 45(3), 180-191.

Berlyne, D. E. (1960). Conflict, arousal, and curiosity. McGraw-Hill Book Company.

Berlyne, D. E. (1966). Curiosity and exploration. Science, 153(3731), 25-33.

Blanchard, T. C., Hayden, B. Y., \& Bromberg-Martin, E. S. (2015). Orbitofrontal cortex uses distinct codes for different choice attributes in decisions motivated by curiosity. Neuron, 85(3), 602-614. 
Bromberg-Martin, E. S., \& Hikosaka, O. (2011). Lateral habenula neurons signal errors in the prediction of reward information. Nature neuroscience, 14(9), 1209-1216.

Cosmides, L., \& Tooby, J. (2000). Evolutionary psychology and the emotions. In M. Lewis, \& J. M. Haviland-Jones (Eds.), Handbook of emotions (2nd ed., pp. 91-115). Guilford.

Csikszentmihalyi, M. (1990). Flow: The Psychology of Optimal Experience. Harper Collins.

Dubey, R., \& Griffiths, T. L. (2020a). Reconciling novelty and complexity through a rational analysis of curiosity. Psychological Review, 127(3), 455-476.

Dubey, R., \& Griffiths, T. L. (2020b). Understanding exploration in humans and machines by formalizing the function of curiosity. Current Opinion in Behavioral Sciences, 35, 118-124.

Dubey, R., Griffiths, T., \& Lombrozo, T. (2019). If it's important, then I am curious: A value intervention to induce curiosity. In CogSci (pp. 282-288).

Farroni, T., Johnson, M. H., Menon, E., Zulian, L., Faraguna, D., \& Csibra, G. (2005). Newborns' preference for face-relevant stimuli: Effects of contrast polarity. Proceedings of the National Academy of Sciences, 102(47), 17245-17250.

Glickman, S. E., \& Sroges, R. W. (1966). Curiosity in zoo animals. Behaviour, 26(1), 151-188.

Gopnik, A. (1996). The scientist as child. Philosophy of science, 63(4), 485-514. https://doi.org/10.1086/289970

Gopnik, A. (2020). Childhood as a solution to explore-exploit tensions. Philosophical Transactions of the Royal Society B, 375(1803), 20190502.

Gopnik, A., Griffiths, T. L., \& Lucas, C. G. (2015). When younger learners can be better (or at least more open-minded) than older ones. Current Directions in Psychological Science, 24(2), 87-92. https://doi.org/10.1177/0963721414556653

Gopnik, A., O’Grady, S., Lucas, C. G., Griffiths, T. L., Wente, A., Bridgers, S., ... \& Dahl, R. E. (2017). Changes in cognitive flexibility and hypothesis search across human life history from childhood to adolescence to adulthood. Proceedings of the National Academy of Sciences, 114(30), 7892-7899.

Gosling, S. D., \& John, O. P. (1999). Personality dimensions in nonhuman animals: A cross-species review. Current directions in psychological science, 8(3), 69-75. 
Gruber, M. J., Gelman, B. D., \& Ranganath, C. (2014). States of curiosity modulate hippocampus-dependent learning via the dopaminergic circuit. Neuron, 84(2), 486-496.

Harlow, H. F. (1950). Learning and satiation of response in intrinsically motivated complex puzzle performance by monkeys. Journal of comparative and physiological psychology, 43(4), 289. https://doi.org/10.1037/h0058114

Harlow, H. F., Harlow, M. K., \& Meyer, D. R. (1950). Learning motivated by a manipulation drive. Journal of Experimental Psychology, 40(2), 228-234.

James, W. (1890). The principles of psychology, Vol. 1. Henry Holt and Co.

Johnson, C., \& Wilbrecht, L. (2011). Juvenile mice show greater flexibility in multiple choice reversal learning than adults. Developmental cognitive neuroscience, 1(4), 540551. https://doi.org/10.1016/j.den.2011.05.008

Kaplan, H., Hill, K., Lancaster, J., \& Hurtado, A. M. (2000). A theory of human life history evolution: Diet, intelligence, and longevity. Evolutionary Anthropology, 9(4), 156-185.

Kashdan, T. B. (2012). Reconsidering the neuroevolutionary framework of the SEEKING system: Emphasizing context instead of positivity. Neuropsychoanalysis, 14(1), 46-50. https://doi.org/10.1080/15294145.2012.10773686

Kashdan, T. B., Disabato, D. J., Goodman, F. R., \& McKnight, P. E. (2020). The FiveDimensional Curiosity Scale Revised (5DCR): Briefer subscales while separating overt and covert social curiosity. Personality and Individual Differences, 157, 109836. https://doi.org/10.1016/j.paid.2020.109836

Kashdan, T. B., Stiksma, M. C., Disabato, D. J., McKnight, P. E., Bekier, J., Kaji, J., \& Lazarus, R. (2018). The five-dimensional curiosity scale: Capturing the bandwidth of curiosity and identifying four unique subgroups of curious people. Journal of Research in Personality, 73, 130-149. https://doi.org/10.1016/j.jrp.2017.11.011

Kidd, C., \& Hayden, B. Y. (2015). The Psychology and Neuroscience of Curiosity. Neuron, 88(3), 449-460.

Kidd, C., Piantadosi, S. T., \& Aslin, R. N. (2012). The Goldilocks effect: human infants allocate attention to visual sequences that are neither too simple nor too complex. PloS One, 7(5), e36399. 
Kidd, C., Piantadosi, S. T., \& Aslin, R. N. (2014). The Goldilocks effect in infant auditory attention. Child Development, 85(5), 1795-1804.

Kobayashi, K., \& Hsu, M. (2019). Common neural code for reward and information value. Proceedings of the National Academy of Sciences, 116(26), 13061-13066.

Levy, D. J., \& Glimcher, P. W. (2012). The root of all value: a neural common currency for choice. Current Opinion in Neurobiology, 22(6), 1027-1038.

Litman, J. A. (2008). Interest and deprivation factors of epistemic curiosity. Personality and individual differences, 44(7), 1585-1595. https://doi.org/10.1016/j.paid.2008.01.014

Litman, J. A., \& Jimerson, T. L. (2004). The measurement of curiosity as a feeling of deprivation. Journal of personality assessment, 82(2), 147-157. https://doi.org/10.1207/s15327752jpa8202_3

LoBue, V., \& DeLoache, J. S. (2008). Detecting the snake in the grass: Attention to fearrelevant stimuli by adults and young children. Psychological science, 19(3), 284-289. https://doi.org/10.1111\%2Fj.1467-9280.2008.02081.x

Loewenstein, G. (1994). The psychology of curiosity: A review and reinterpretation. Psychological bulletin, 116(1), 75. https://doi.org/10.1037/0033-2909.116.1.75

Lucas, C. G., Bridgers, S., Griffiths, T. L., \& Gopnik, A. (2014). When children are better (or at least more open-minded) learners than adults: developmental differences in learning the forms of causal relationships. Cognition, 131(2), 284-299.

Marr, D. (1982). Vision: A computational investigation into the human representation and processing of visual information. Freeman

Montag, C., \& Panksepp, J. (2017). Primary Emotional Systems and Personality: An Evolutionary Perspective. Frontiers in Psychology, 8, 464.

O’Hara, M., \& Auersperg, A. M. (2017). Object play in parrots and corvids. Current opinion in behavioral sciences, 16, 119-125. https://doi.org/10.1016/j.cobeha.2017.05.008

Öhman, A., \& Mineka, S. (2003). The malicious serpent: Snakes as a prototypical stimulus for an evolved module of fear. Current directions in psychological science, 12(1), 5-9. https://doi.org/10.1111\%2F1467-8721.01211

Oosterwijk, S. (2017). Choosing the negative: A behavioral demonstration of morbid 
curiosity. PloS One, 12(7), e0178399.

Panksepp, J., \& Moskal, J. (2008). Dopamine and SEEKING: Subcortical "reward" systems and appetitive urges. In A. J. Elliot (Ed.), Handbook of approach and avoidance motivation (p. 67-87). Psychology Press. https://doi.org/10.4324/9780203888148.ch5

Parker, K. J., Rainwater, K. L., Buckmaster, C. L., Schatzberg, A. F., Lindley, S. E., \& Lyons, D. M. (2007). Early life stress and novelty seeking behavior in adolescent monkeys. Psychoneuroendocrinology, 32(7), 785-792.

Perry, S. E., Barrett, B. J., \& Godoy, I. (2017). Older, sociable capuchins (Cebus capucinus) invent more social behaviors, but younger monkeys innovate more in other contexts. Proceedings of the National Academy of Sciences, 114(30), 7806-7813. https://doi.org/10.1073/pnas.1620739114

Piaget, J. (1945). Play, Dreams and Imitation in Childhood. Psychology Press.

Reader, S. M. (2015). Causes of Individual Differences in Animal Exploration and Search. Topics in Cognitive Science, 7(3), 451-468.

Schrock, J. M., Snodgrass, J. J., \& Sugiyama, L. S. (2020). Lassitude: The emotion of being sick. Evolution and Human Behavior, 41(1), 44-57. https://doi.org/10.1016/j.evolhumbehav.2019.09.002

Scrivner, C. (2020). The Psychology of Morbid Curiosity. Psyarxiv. https://doi.org/10.31234/osf.io/xug34

Scrivner, C. (2021). An Infectious Curiosity: Morbid Curiosity and Media Preferences During a Pandemic. Evolutionary Studies in Imaginative Culture, 5(1).

Scrivner, C., Johnson, J. A., Kjeldgaard-Christiansen, J., \& Clasen, M. (2021). Pandemic practice: Horror fans and morbidly curious individuals are more psychologically resilient during the COVID-19 pandemic. Personality and Individual Differences, 168, 110397.

Scrivner, C., \& Maestripieri, D. (2018). Creativity Patterns in the Production of Scientific Theories and Literary Fiction. KNOW: A Journal on the Formation of Knowledge, 2(1), 137-154. https://doi.org/10.1086/696984

Shin, D. D., \& Kim, S. I. (2019). Homo curious: Curious or interested?. Educational Psychology Review, 1-22. https://doi.org/10.1007/s10648-019-09497-x

Sih, A., Bell, A. M., Johnson, J. C., \& Ziemba, R. E. (2004). Behavioral syndromes: an 
integrative overview. The quarterly review of biology, 79(3), 241-277.

https://doi.org/10.1086/422893

Silvia, P. J. (2010). Confusion and interest: The role of knowledge emotions in aesthetic experience. Psychology of Aesthetics, Creativity, and the Arts, 4(2), 75. https://doi.org/10.1037/a0017081

Sznycer, D., Cosmides, L., \& Tooby, J. (2017). Adaptationism carves emotions at their functional joints. Psychological Inquiry, 28(1), 56-62.

https://doi.org/10.1080/1047840X.2017.1256132

Tinbergen, N. (1963). On aims and methods of ethology. Zeitschrift für tierpsychologie, 20(4), 410-433. https://doi.org/10.1111/j.1439-0310.1963.tb01161.x

Wang, M. Z., \& Hayden, B. Y. (2019). Monkeys are curious about counterfactual outcomes. Cognition, 189, 1-10.

Wright, J. S., \& Panksepp, J. (2012). An evolutionary framework to understand foraging, wanting, and desire: the neuropsychology of the SEEKING system. Neuropsychoanalysis, 14(1), 5-39. https://doi.org/10.1080/15294145.2012.10773683

Yee, M., Jones, S. S., \& Smith, L. B. (2012). Changes in visual object recognition precede the shape bias in early noun learning. Frontiers in Psychology, 3, 533. 